\title{
Procedimiento EXIT (ex utero intrapartum) en las malformaciones congénitas broncopulmonares de alto riesgo. Descripción de la técnica y revisión de la literatura
}

\author{
Rogelio Sancho-Hernández,*,*, $₫$ Lizbeth Solorio-Rodríguez,* \\ Anné Azriel Durán-Colín,* Francisco Javier Cuevas-Schacht*
}

*Hospital Materno Perinatal Mónica Pretelini Sáenz, Ciudad de Toluca, Estado de México;

Instituto Nacional de Pediatría, Ciudad de México.

Trabajo recibido: 22-VI-2016; aceptado: 05-X-2016

\begin{abstract}
RESUMEN. Introducción: La ultrasonografía prenatal representa en la actualidad una forma de presentación de las malformaciones congénitas broncopulmonares (MCBP), estas lesiones intratorácicas pueden ocasionar polihidramnios, desviación mediastinal, hidropesía y muerte fetal. Se presenta el primer reporte nacional y se describe la técnica del procedimiento EXIT (ex utero intrapartum) como opción para estas masas pulmonares fetales de alto riesgo. Descripción de casos: Feto de $30 \mathrm{SG}$ con una masa microquística de todo el pulmón izquierdo, con desviación mediastinal e hidropesía fetal, con un CVR de 2.7. El embarazo fue llevado a término y se realizó procedimiento EXIT con apoyo de flujo útero-placentario exitoso de 63 minutos con intubación selectiva de bronquio derecho por broncoscopia rígida y neumonectomía izquierda de una lesión sólida compatible con MAQ tipo I difusa y focos aislados de tipo 0 . El paciente falleció por complicaciones asociadas al barotrauma y a la hipoplasia e hipertensión pulmonar refractarias a manejo convencional. Discusión: El procedimiento EXIT puede representar en nuestro medio una opción terapéutica para las MCBP de alto riesgo con desviación mediastinal persistente próximos al término del nacimiento. La afección difusa del pulmón afectado, la hipoplasia pulmonar contralateral y la hipertensión pulmonar refractarias continúan siendo los factores que definen la mortalidad perinatal.
\end{abstract}

Palabras clave: EXIT, ex utero intrapartum, masas pulmonares fetales, CVR.

ABSTRACT. Introduction: Prenatal ultrasonographic now represents a form of presentation of congenital bronchopulmonary malformations (CBPM), these intrathoracic lesions can cause polyhydramnios, mediastinal shift, hydrops and fetal death, it presents the first national report and process engineering EXIT described (ex utero intrapartum) as an option for these fetal lung masses high risk. Description of cases: Fetus 30 SG with a microcystic mass of the entire left lung with mediastinal shift and fetal hydrops, with a CVR 2.7, the pregnancy was carried to term and procedure EXIT is carried out with the support of utero flow successful placental 63 minutes with selective intubation of right bronchus by rigid bronchoscopy and left pneumonectomy a solid lesion compatible with MAQ type I diffuse and isolated pockets of type 0, patient died from pulmonary complications associated with barotrauma and hypoplasia and hypertension refractory to management conventional. Discussion: The EXIT procedure in our area may represent a therapeutic option for high-risk MCBP persistent near-term birth mediastinal deviation; the diffuse involvement of the affected lung, contralateral pulmonary hypoplasia and pulmonary hypertension refractory remain the defining factors perinatal mortality.

Key words: EXIT, ex utero intrapartum, fetal lung masses, CVR.

\section{INTRODUCCIÓN}

La ultrasonografía prenatal ha incrementado el diagnóstico de las malformaciones congénitas broncopulmonares (MCBP), y con ello ha impactado en el tratamiento perinatal y el pronóstico de estas masas pulmonares fetales. ${ }^{1}$ Dichas masas han correspondido con una variedad de subtipos patológicos: malformación adenomatoidea quística (MAQ), secuestro broncopulmonar (SBP), quiste broncogénico, hiperinsuflación lobar congénita, atresia bronquial, y los inusuales blastoma pleuropulmonar (BPP) y tumor pulmonar intersticial; ;-4 la historia natural de las masas pulmonares fetales depende del tamaño y el trastorno fisiológico causado 
por el efecto de compresión a las estructuras adyacentes; ${ }^{2,5}$ en menos del $4 \%$ ocasionan efecto de masa con desviación mediastinal, hidropesía y muerte fetal. ${ }^{6}$ Las opciones de tratamiento para las grandes masas pulmonares fetales incluyen en la gestación temprana la administración de esteroides, la toracocentesis o derivación toracoamniótica intrauterinas y la resección quirúrgica fetal, y para los fetos con masas de alto riesgo al final de la gestación el procedimiento EXIT (ex utero intrapratum) ha sido propuesto. El objetivo de EXIT es mantener la perfusión placentaria para optimizar la transición de la vida fetal a la neonatal: estabilizar la vía aérea comprometida, controlar la desviación mediastinal y compresión cardíaca y limitar las complicaciones asociadas a la hipoplasia con hipertensión pulmonar y la muerte por hidropesía fetal. ${ }^{2,5,7-9}$ La práctica de EXIT para el manejo de estas masas pulmonares fetales es limitada, presentamos el primer reporte nacional y latinoamericano de la experiencia en el procedimiento EXIT para la resección pulmonar de estas masas torácicas de alto riesgo. Se describe la técnica quirúrgica y la revisión de la literatura para orientar las posibles recomendaciones y el estado actual de EXIT en los centros fetales especializados.

\section{Presentación de caso}

Femenino de 20 años, gesta 4, para 3, referida a las 28 semanas de gestación (SG) para la evaluación de masa pulmonar fetal que ocupa todo el hemitórax izquierdo. El ultrasonido fetal muestra una masa hiperecogénica (microquística-sólida) de $5.36 \times 3.79 \times 7.35 \mathrm{~cm}$, recibe vascularidad de la arteria pulmonar izquierda, con desviación mediastinal y del área cardíaca hacia la derecha, el pulmón derecho se aprecia con una ecogenicidad normal de $2.15 \times 2.39 \mathrm{~cm}$, área de $3.67 \mathrm{~cm}^{2}$ y se aprecia líquido de ascitis y escaso líquido pleural sin otros datos de hidropesía fetal, no se identifican otras alteraciones estructurales, ni marcadores para cromosomopatías detectables, se calculó por fetometría $30 \mathrm{SG}$ con peso fetal estimado de 1,589 g, placenta corporal anterior lateral izquierda. La madre recibió un ciclo corto de esteroide (dosis diaria de betametasona $12.5 \mathrm{mg}$ IM en dos días consecutivos), una última valoración ultrasonográfica mostró a las 31.2 SG feto con probable MAQ microquística-sólida tipo III de todo el pulmón izquierdo, con peso fetal estimado en 1,714 g y remisión de la ascitis y derrame pleural fetales, con un CVR de 2.7 (relación volumen de lesión/circunferencia cefálica fetal de 2.7, mayor de 1.6 como límite de referencia), persiste con la compresión cardíaca y desviación mediastinal, pulmón derecho con un cociente pulmón: cabeza de 0.13 . Se tomaron muestras de cariotipo, se solicitó resonancia magnética fetal y se propuso manejo multidisciplinario (Neonatología, Genética, Medicina Materno Fetal, Cirugía de Tórax Pediátrica, Anestesiología, Cardiología, Enfermería, Psicología y Comité de Ética) para candidato a procedimiento EXIT y posible resección de masa pulmonar fetal de alto riesgo por refractariedad al manejo conservador y alta posibilidad de muerte al nacer (figura 1).

\section{Descripción de la técnica}

Las indicaciones para el nacimiento por vía EXIT fueron la presencia de una masa fetal de alto riesgo con severa desviación mediastinal y compresión cardíaca asociados a un persistente aumento en el CVR fetal (a pesar de la remisión de la hidropesía fetal por los esteroides maternos), así como el no ser candidato a otras técnicas conservadoras por la naturaleza sólida de la masa fetal (toracocentesis, derivación toraco-amniótica y embolización en ausencia de vaso aberrante por SBP).

La madre recibió un curso de betametasona en el perioperatorio, previa inducción anestésica obstétrica; y una vez que la relajación uterina fue confirmada y la posición placentaria corroborada se procedió a realizar la histerotomía a $5 \mathrm{~cm}$ del margen placentario bajo guía ultrasonográfica, se realizó el parto parcial con la liberación y exposición de la cabeza, brazos y tórax fetal (cuidando de evitar la exposición abdominal y compresión de cordón umbilical) y se procedió a realizar una broncoscopia rígida neonatal con equipo de $3.5 \mathrm{~mm}$ donde se aprecia desviación hacia la derecha y colapso total de la carina y compresión anterolateral izquierda de la tráquea distal, se avanza el equipo hasta la emergencia del bronquio derecho y bajo guía metálica con técnica de Seldinger se realiza una intubación selectiva del bronquio derecho y estabilización de la vía aérea con cánula endotraqueal de $3.5 \mathrm{~mm}$ la cual es fijada con un punto de sutura al labio superior del feto. En el trascurso de todo el procedimiento EXIT la ventilación fue evitada para preservar la circulación fetal-placentaria y obviar la expansión del pulmón izquierdo afectado por la MAQ sólida (figura 2). De manera coordinada el equipo multidisciplinario procedió a canalizar un acceso venoso y oximetría de pulso de la mano izquierda fetal y colocar el transductor torácico para la monitorización ecocardiográfica, al mismo tiempo el volumen uterino es mantenido por una infusión continua de solución Hartmann con la finalidad de evitar el desprendimiento placentario y permitir la circulación feto-placentaria, una vez asegurado este primer punto crítico con estabilización de la vía aérea, acceso venoso seguro y monitorización fetal con oximetría y ecocardiografía (FC de 167 pm, saturación fetal de 36\% y función sistólica-diastólica conservadas) el feto recibió 

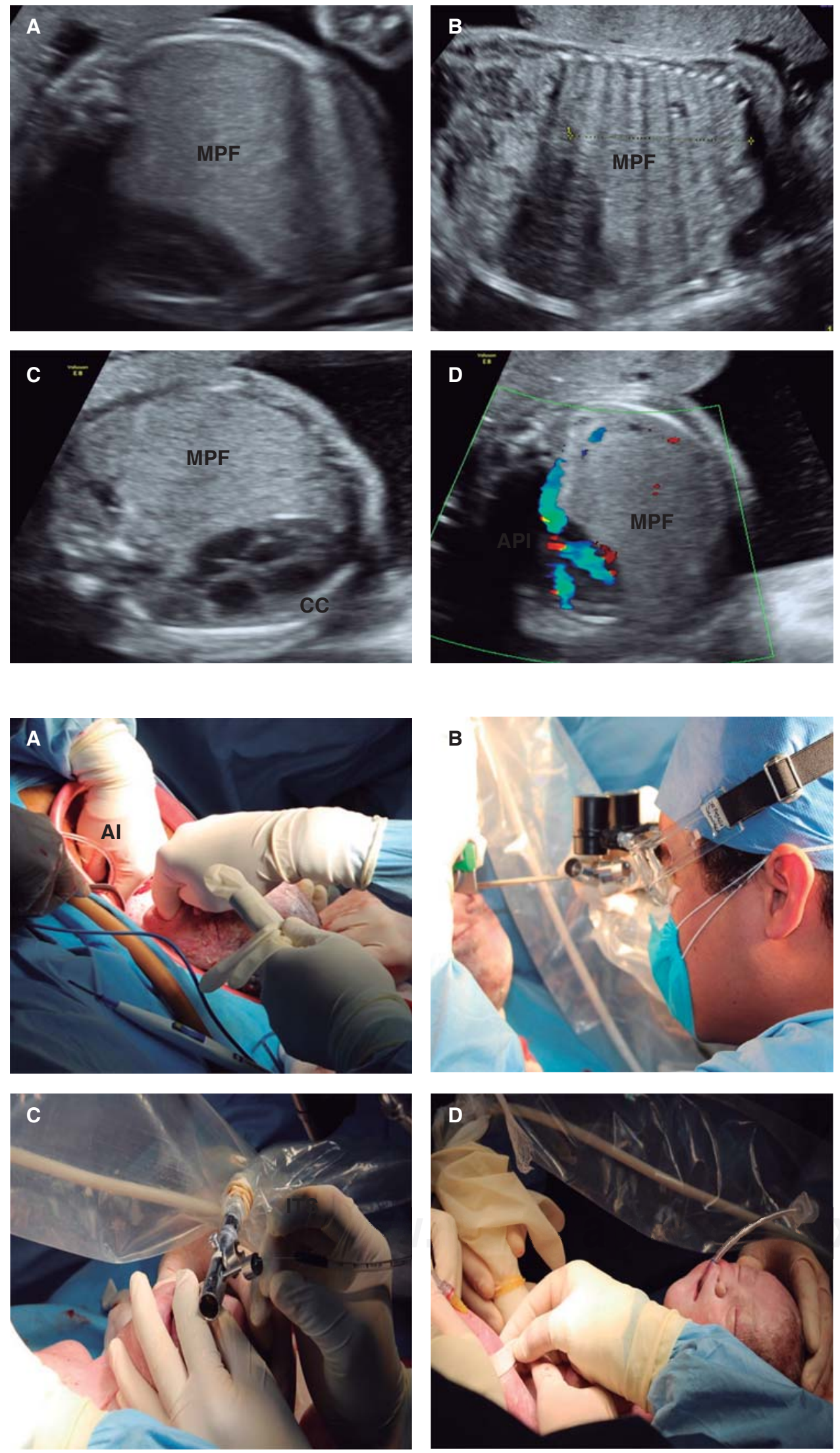

Figura 1.

En A: masa pulmonar fetal (MPF) sólida-microquística de todo el hemitórax izquierdo a las 28 SG; en $\mathbf{B}$ : derrame pleural escaso y líquido de ascitis compatible con hidropesía fetal; en C: reversión de la hidropesía con ciclo de esteroides maternos pero persiste la desviación mediastinal y compresión cardíaca (CC) por la MPF a las 31 SG; en D: irrigación de la MPF por la arteria pulmonar izquierda (API) con aumento persistente del CVR hasta 2.7, compatible prenatalmente con probable MAQ tipo III.

\section{Figura 2.}

En A: parto parcial con inicio de la amnioinfusión (Al); en B: diagnóstico de la vía aérea comprometida a través de la broncoscopia rígida; en C: intubación endotraqueal selectiva del bronquio principal derecho con técnica de Seldinger (ITS) bajo guía metálica para estabilizar la vía aérea; en D: cánula endotraqueal fijada, se evita la ventilación durante todo el procedimiento EXIT para preservar la circulación útero-placentaria. 
una dosis de fentanil $20 \mu \mathrm{g} / \mathrm{kg}$, vecuronio $200 \mu \mathrm{g} / \mathrm{kg}$ y atropina $20 \mu \mathrm{g} / \mathrm{kg}$, después se colocó al feto en decúbito lateral derecho para iniciar la toracotomía posterolateral izquierda (figura 3).

Al ejecutar la toracotomía, la ecocardiografía advierte en este segundo punto crítico remisión de la compresión cardíaca y centralización del mediastino, la cual se correlaciona con una FC de 168 pm y elevación de la saturación fetal a $54 \%$, por lo que con esta estabilidad hemodinámica se procedió a realizar la resección de toda la masa sólida, pétrea, nodular y microquística a través de una neumonectomía izquierda; al momento del control vascular y posterior ligadura de la arteria pulmonar izquierda se advierte en un tercer punto crítico ascenso de la saturación fetal a $65 \%$ y FC de $170 \mathrm{Ipm}$ correlacionando el comportamiento de cortocircuito vascular-pulmonar generado por la MAQ sólida (el rango normal de saturación arterial de oxígeno en el feto se encuentra entre el 30-70\%), (figura 4).

\section{Manejo posnatal}

Una vez insertada una sonda intratorácica para evaluar la presencia de hemorragia o fuga aérea fue cerrada la toracotomía izquierda, se pinzó y cortó el cordón umbilical y la ventilación convencional (VC) fue iniciada y el neonato de término estabilizado bajo la monitorización ecocardiográfica, no se administró surfactante. Bajo estabilidad hemodinámica se iniciaron parámetros basales de VC con presión inspiratoria de 18 y presión positiva al final de la espiración de 4 para mantener saturación del 94\% por oximetría; se requirió de incrementos en parámetros ventilatorios y el neonato complica con volu-barotrauma a los 30 minutos del nacimiento, lo cual requiere la inserción de una sonda pleural derecha para revertir el neumotórax a tensión y se advierten datos radiológicos de hipoplasia pulmonar residual derecha, se presenta hipotensión y desaturación y la ecocardiografía demuestra una medición indirecta de la presión de arteria pulmonar en $50 \mathrm{mmHg}$ con lo que se inicia terapia farmacológica con vasodilatadores pulmonares e inotrópicos y se instaura la ventilación de alta frecuencia oscilatoria (VAFO), prosigue con los datos de cortocircuito vascular-pulmonar y se inicia a las 2 horas de vida neonatal la administración de óxido nítrico a 20 partículas por millón. Con una estabilidad relativa presenta a las 6 horas de vida un evento de paro cardiorrespiratorio que no responde a las maniobras convencionales de reanimación avanzada (figura 5A).

En la evaluación histopatológica se advierte una pieza quirúrgica producto de neumonectomía izquierda de apariencia y consistencia sólida, con dilatación bronquiolar terminal, fibrosis y hemorragia perialveolar compatible con MAQ tipo I difusa, al identificarse células caliceales propias de la vía aérea mayor se sugirieron componentes de MAQ tipo 0 , hallazgos potencialmente incompatibles con la vida y función (figura $5 \mathrm{~B}, \mathrm{C}$ y D).

\section{DISCUSIÓN}

El procedimiento EXIT fue en su origen descrito para retirar el balón de oclusión traqueal en los fetos con

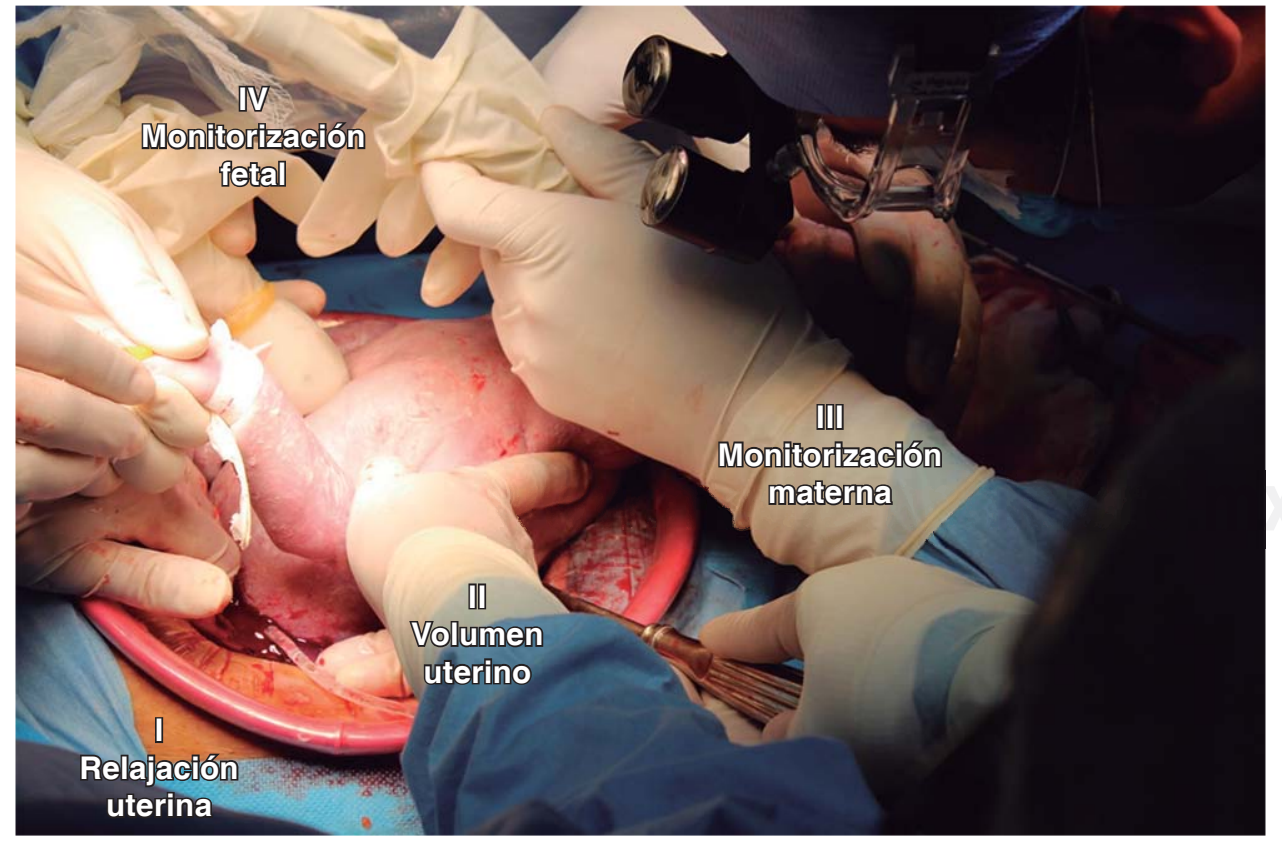

Figura 3.

Elementos del EXIT integrados en el primer paso crítico: I. Relajación uterina (anestesia inhalada y tocolíticos) para la preservación del flujo útero-placentario; en II. Volumen uterino conservado a través de la Al; en III. Monitorización materna (TA invasiva, EKG continua, $\mathrm{O}_{2}$ pulso y $\mathrm{CO}_{2}$ tidal); en IV. Monitorización fetal (acceso venoso y anestesia fetal, $\mathrm{O}_{2}$ pulso y ecocardiografía). Se asegura la vía aérea comprometida con apoyo de broncoscopia rígida y la coordinación simultánea de un equipo multidisciplinario. 

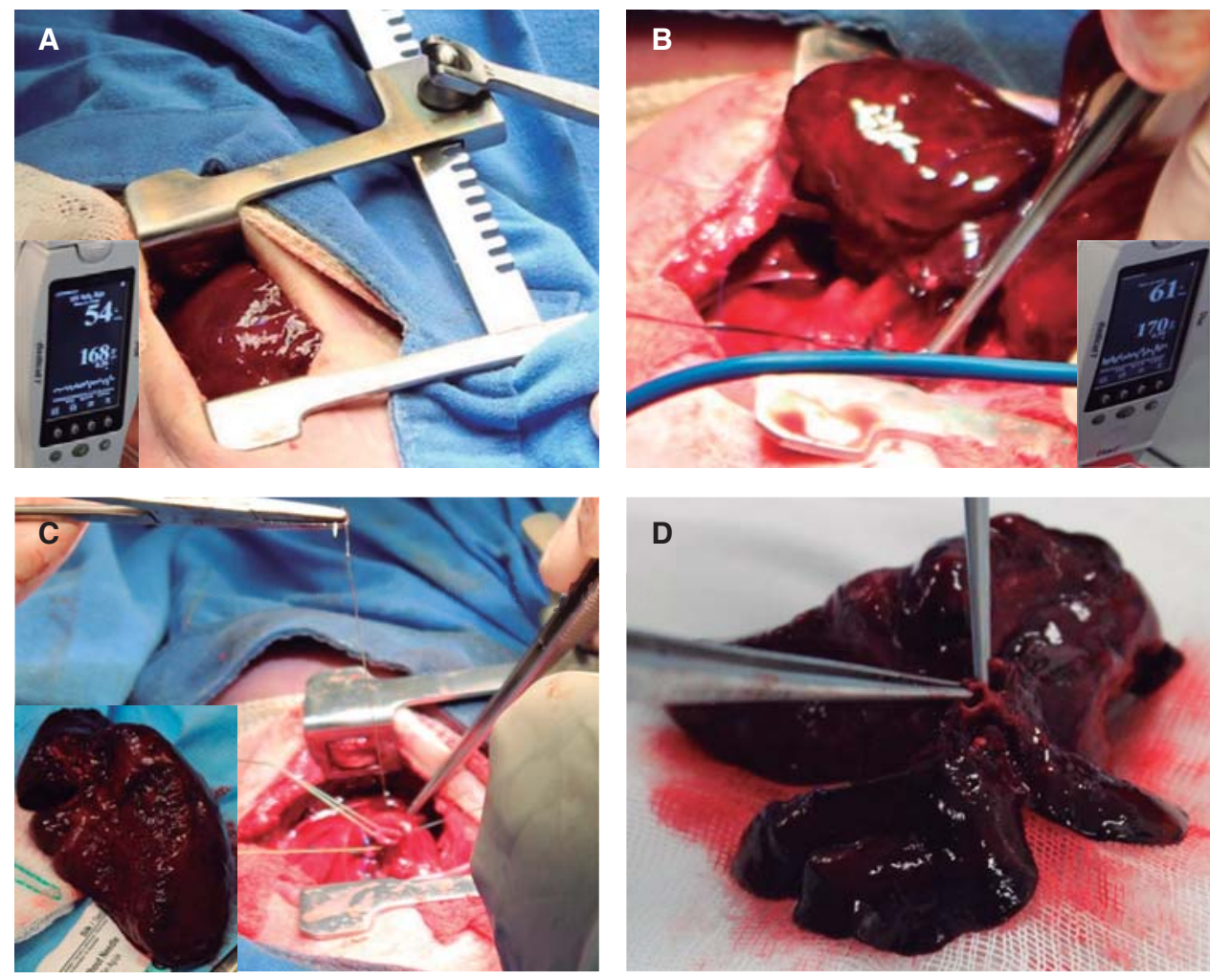

Figura 4.

EXIT durante el segundo punto crítico: en $\mathbf{A}$, la toracotomía se correlaciona en la ecocardiografía con reversión de la compresión cardíaca y centralización del mediastino; en $\mathbf{B}$, el tercer punto crítico: ascenso de la saturación fetal a $65 \%$ y FC de 170 Ipm posterior al pinzamiento de la arteria pulmonar; en $\mathbf{C}$, resección de la masa sólida por neumonectomía y cierre del muñón bronquial; en D, disminución del calibre del bronquio principal izquierdo y apariencia sólido-quística de la masa fetal. hernia diafragmática severa quienes habían sido candidatos a oclusión traqueal fetoscópica con balón. El uso del flujo útero-placentario concede un tiempo quirúrgico para retirar durante el parto parcial el balón traqueal, estabilizar la vía aérea comprometida, administración de surfactante y fármacos de resucitación y establecer un acceso venoso y arterial en estos neonatos inestables por la hipoplasia e hipertensión pulmonar severas. ${ }^{1,10}$

Recién las indicaciones de EXIT se han extendido a otros procedimientos clasificados con base en el tipo de lesión y región fetal comprometidas:

a) EXIT para estabilizar la vía aérea: obstrucción por masas cervicales (teratoma cervical, malformación vascular-linfática) y síndromes obstructivos de la vía aérea superior (CHAOS: atresia laríngea y traqueal, micrognatia en síndrome de Pierre-Robin, etc).

b) EXIT para procedimiento de resección: masas intratorácicas fetales con obstrucción de la vía aérea: teratoma mediastinal y pericárdico y malformaciones congénitas broncopulmonares de alto riesgo como en nuestro caso reporte.

c) EXIT para procedimiento ECMO (oxigenación por membrana extracorpórea): hernias diafragmáticas severas, cardiopatía congénita severa y masas intratorácicas fetales de alto riesgo. d) EXIT para procedimiento de separación: gemelos unidos..$^{11,12}$

Podemos apreciar que la mayoría de estas indicaciones se incluyen dentro de las repercusiones fisiopatológicas de las masas broncopulmonares de alto riesgo, en donde la compresión esofágica por la masa torácica puede interferir con la deglución y circulación de líquido amniótico fetal y resultar en polihidramnios, ${ }^{13}$ podrían causar extrema desviación mediastinal con compresión cardíaca y de la vena cava, como en nuestro reporte, y evolucionar a la hidropesía con muerte fetal y posnatal inmediata, ${ }^{14}$ este efecto de masa también impacta en la hipoplasia e hipertensión vascular y pulmonar secundarias y en la función comprometida del pulmón residual y contralateral. En el momento del nacimiento estas MAQ sólidas fetales podrían complicar la estabilidad cardiorrespiratoria al experimentar sobredistensión de las lesiones quísticas pulmonares posterior a la primera inspiración o a la ventilación con presión positiva al momento de instaurarse el cuadro de dificultad respiratoria e inestabilidad de la vía aérea durante la reanimación neonatal., ${ }^{1,2}$

Hacia la década de los noventa con el advenimiento de la ultrasonografía prenatal se empezaron a correlacionar estas masas pulmonares sólidas y quísticas con los productos de hidropesía e hipoplasia pulmonar 

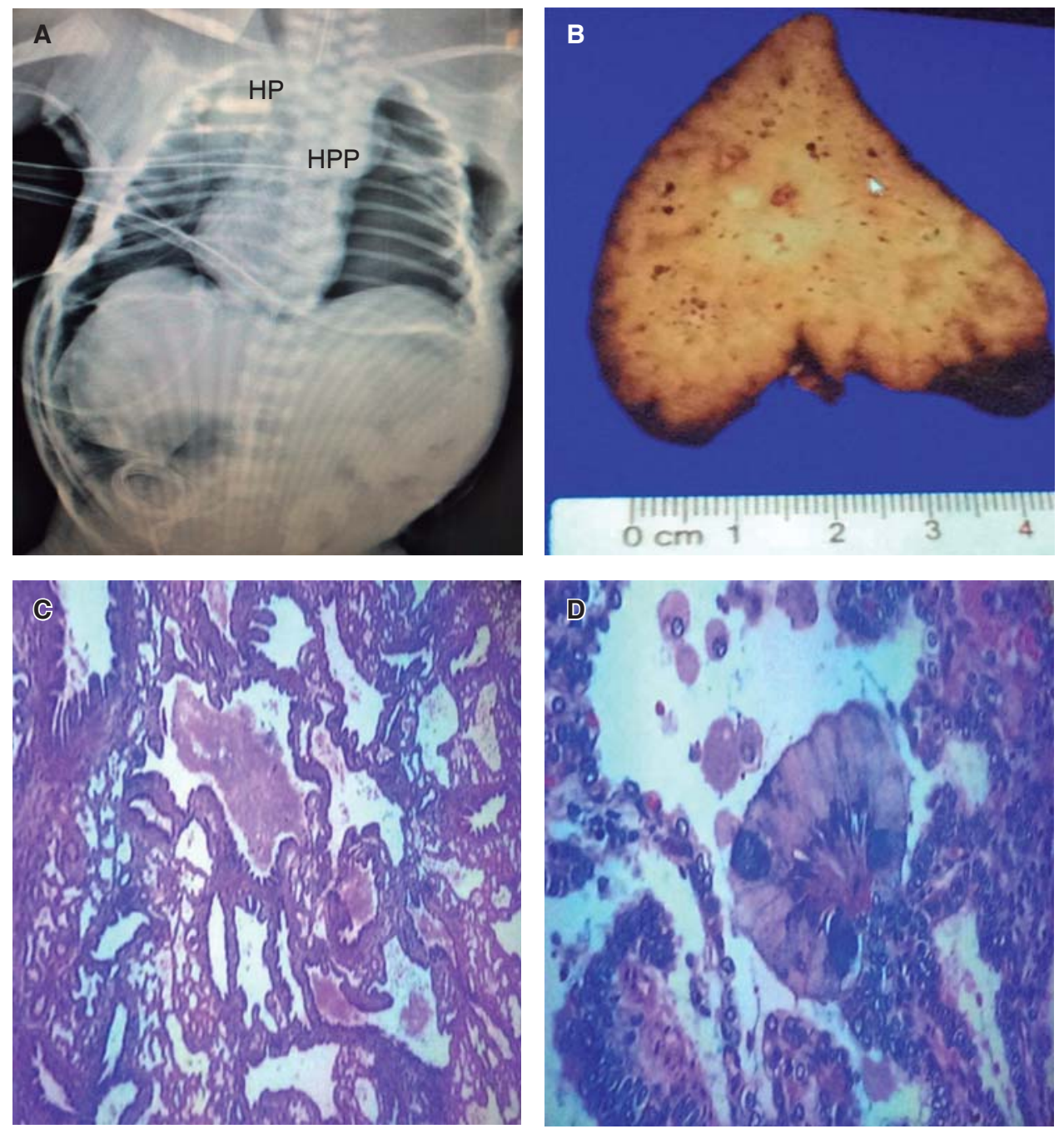

\section{Figura 5.}

En A: neumotórax a tensión y neumoretroperitoneo secundario a volu-barotrauma del pulmón hipoplásico residual (HP), se insertaron dos sondas pleurales, muerte por hipoplasia pulmonar e hipertensión pulmonar persistente (HPP) refractaria a manejo convencional; en B: patología macroscópica con pieza quirúrgica notablemente sólida; en $\mathbf{C}$ : dilatación bronquiolar terminal, fibrosis y hemorragia perialveolar compatible con MAQ tipo I difusa; y en D: célula caliceal sugerente de focos de MAQ tipo 0. de las autopsias fetales y de recién nacidos fallecidos con dificultad respiratoria e hipertensión pulmonar persistentes. El análisis patológico reveló desde entonces las nuevas formas de presentación prenatal de los SBP y de las MAQ. ${ }^{15}$ Adzick et al. clasificaron las MAQ prenatales en dos categorías basados en su anatomía macroscópica y los hallazgos ultrasonográficos: macroquísticas las potenciales MAQ que contenía un único quiste predominante o múltiples quistes $>5 \mathrm{~mm}$ de diámetro con apariencia notablemente quística, y microquísticas las lesiones con quistes < de $5 \mathrm{~mm}$ y de apariencia sólida o notablemente ecogénicas como nuestro caso. ${ }^{16}$ Entonces se esperaba que las lesiones macroquísticas prenatales correspondieran con los tipos posnatales I y II de Stocker y las microquísticas con la tipo III, pero sorprende una pobre correlación entre los diagnósticos prenatales ultrasonográficos; y los posnatales histopatológicos agregaron nuevos diagnósticos hasta en $8.6 \%$ de estas masas fetales y las lesiones microquísticas empezaron a corresponder con MAQ tipo III, neuroblastoma fetal y SBP extralobar, y los macroquísticos con MAQ tipo I y II, quistes broncogénicos y neuroentéricos, con los SBP intralobares e inclusive con las lesiones híbridas con componentes mixtos de MAQ y SBP. ${ }^{6,17-20}$ Esta controversia la podemos constatar en nuestro caso con el reporte prenatal de masa torácica microquística-sólida que correspondió divergentemente con los hallazgos histopatológicos de MAQ tipo 0 y l. Hemos aprendido desde entonces que el pronóstico de las masas fetales intratorácicas depende primariamente del tamaño más que del tipo de la lesión y que reconocer las características de su crecimiento resultaría importante para entender la historia natural polimorfa de las MCBP.

Con el objeto de identificar un parámetro prenatal con facultad para predecir el comportamiento de las masas fetales de alto riesgo que evolucionarían a la hidropesía fetal y a la dificultad respiratoria al nacer se determinó 
el CVR: relación volumen de lesión/circunferencia cefálica fetal, este volumen se obtiene de la medición ultrasonográfica de las dimensiones de la lesión MAQ aplicadas al cálculo de la elipse: $\mathbf{C V R}=$ (longitud $\times$ altura $\times$ ancho $\times 0.523$ en $\mathrm{cm}^{3}$ )/circunferencia cefálica fetal, se determinó que un CVR $\geq 1.6$ correlacionaba con alto riesgo para desarrollar hidropesía y muerte fetal, necesidad de intervención prenatal, necesidad de soporte ventilatorio, necesidad de ECMO, predictor de la sintomatología respiratoria y hemodinámica al nacer, de la estancia intrahospitalaria y la sobrevida posnatal inmediata. ${ }^{21,22}$ En nuestro reporte podemos advertir un CVR de 2.7 persistente en la evaluación ultrasonográfica semanal con desviación mediastinal y compresión cardíaca, refractario a la intervención con esteroides maternos y que de acuerdo con las recomendaciones de los centros fetales estos pacientes con pobre pronóstico serían candidatos a la estabilización y resección de su masa pulmonar por la vía del procedimiento EXIT ${ }^{1,16}$ (figura 6).

El principio central de EXIT es controlar la hipotonía uterina que permita la circulación útero-placentaria, mantener la presión sanguínea materna es obligatorio para asegurar una adecuada oxigenación fetal durante el procedimiento: el gasto cardíaco del feto depende de la frecuencia cardíaca más que del volumen sistólico y la saturación arterial fetal normal oscila entre 40 y $70 \%$. Esta estabilidad transoperatoria se pudo alcanzar bajo la vigilancia ecocardiográfica de la frecuencia cardíaca, contractilidad miocárdica, constricción ductal y competencia valvular aurículo ventricular íntegras, la evaluación integral identifica los signos de dificultad circulatoria fetal que requerirán la administración de líquidos, hemoderivados y fármacos inotrópicos por un acceso venoso previamente asegurado. El procedimiento EXIT se integra de cuatro elementos fisiológicos para garantizar el flujo útero-placentario: a) relajación uterina a través de la anestesia inhalada obstétrica y los agentes tocolíticos, ambos preservan la perfusión placentaria; b) mantenimiento del volumen uterino por medio de la amniotransfusión continua con solución Hartmann y el parto parcial, ambos previenen la contracción uterina y en consecuencia preservan el flujo placentario; c) monitorización fetal con la oximetría de pulso y la ecocardiografía, y d) monitorización materna a través de la tensión arterial invasiva, electrocardiograma continuo, oximetría de pulso y $\mathrm{CO}_{2}$ al final de la espiración. La coordinación de todos estos elementos por parte del equipo multidisciplinario puede observarse en la descripción previa de la técnica quirúrgica, las altas concentraciones del anestésico inhalado para proveer la relajación uterina también pueden incrementar el riesgo potencial de hemorragia materna y riesgo de histerectomía. En nuestros pacientes no existieron complicaciones durante el procedimiento EXIT, previo al pinzamiento del cordón umbilical esta coordinación es elemental para restaurar el tono uterino mediante la disminución de los anestésicos inhalados y el inicio

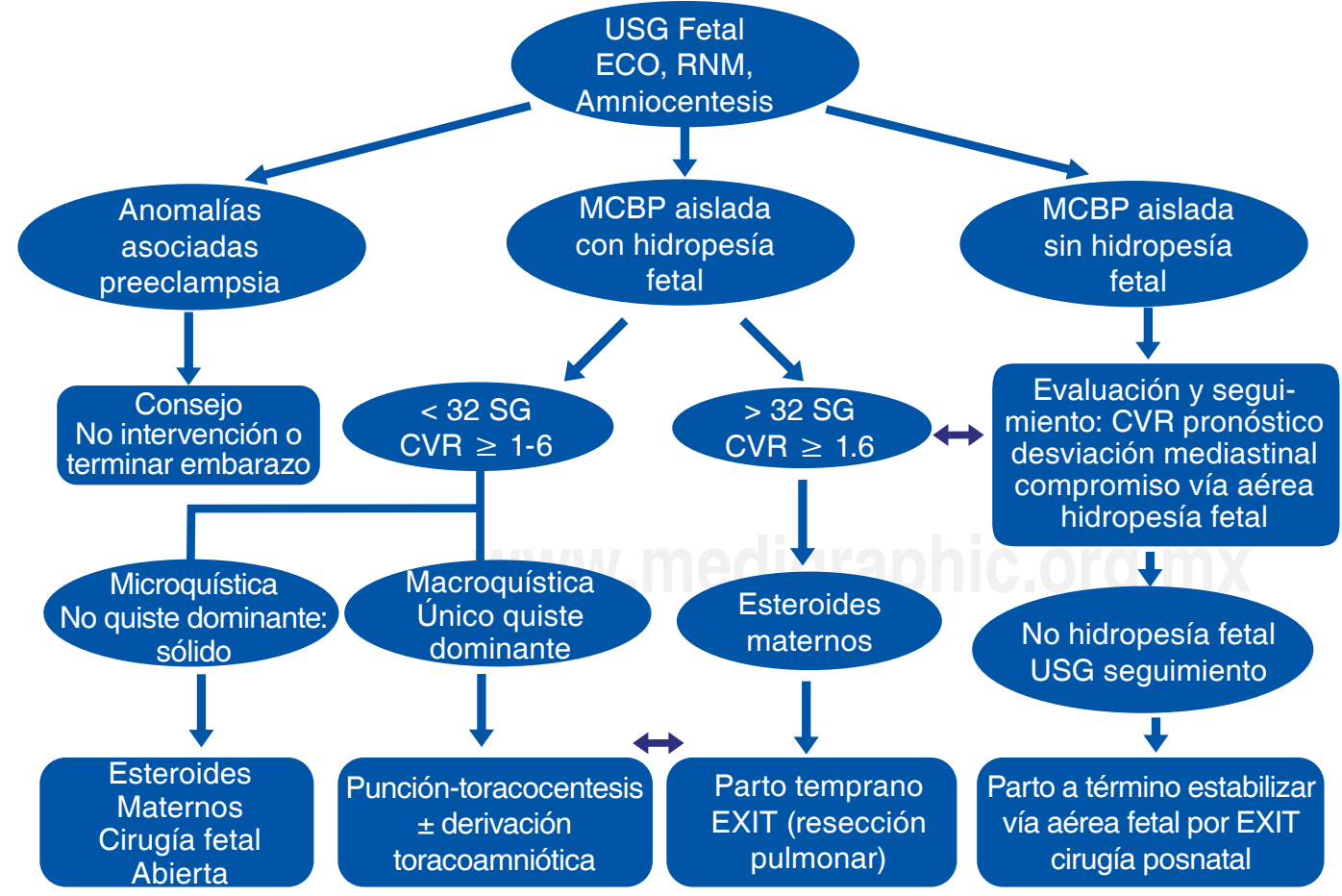

Figura 6.

Algoritmo para el diagnóstico y tratamiento perinatal de las masas intratorácicas y malformaciones congénitas broncopulmonares fetales. 
de la infusión de oxitocina que prevenga la hemorragia materna. En el feto las potenciales complicaciones durante el EXIT están relacionadas a la falla para preservar el intercambio gaseoso útero-placentario por la compresión del cordón umbilical, el desprendimiento placentario y la pérdida de la relajación uterina; ${ }^{23,24}$ asegurada la perfusión útero-placentaria se pueden practicar en un período de tiempo procedimientos quirúrgicos y endoscópicos seguros en el feto. En nuestro caso se optó primero en asegurar y estabilizar una vía aérea crítica comprometida por el efecto de masa, se ha descrito la laringoscopia con intubación endotraqueal convencional y en otros, como en nuestro reporte, ejecutar una broncoscopia rígida con una intubación bajo guía metálica con técnica de Seldinger a través de la cual se transporta la cánula endotraqueal armada al bronquio selecto (figura 2). La broncoscopia flexible cuando idealmente se cuente con ella complementa las ventajas del procedimiento como para apoyar una traqueostomía o proteger la disección de masas cervicales con dificultades para la intubación o que se conviertan en candidatos para la resección misma durante el EXIT.

En ocasiones es necesario descomprimir el componente quístico de la masa cervical, mediastinal o torácica bajo guía ultrasonográfica con ayuda de una aguja o punzocat espinal para asegurar la vía aérea; la perfusión útero-placentaria ha permitido elaborar procedimientos en $90 \mathrm{~min}$; en nuestro reporte la resección pulmonar fue posible en $63 \mathrm{~min} .{ }^{25}$ No obstante se empiezan a sumar reportes en torno a la canulación venosa-arterial cervical (yugular interna-carótida) para la inducción de ECMO-EXIT en masas pulmonares fetales, cardiopatías complejas y, más controversial, en las hernias diafragmáticas de alto riesgo. ${ }^{26,27}$

La resección de masas pulmonares de alto riesgo con apoyo de EXIT logró en una revisión de 9 pacientes con promedio de 35.4 SG y con CVR promedio de 2.2 una sobrevida del $89 \%$, el tiempo promedio de perfusión placentaria fue de $65 \mathrm{~min}, 6$ fetos recibieron resucitación hídrica, transfusional e inotrópica y 4 neonatos requirieron de ECMO como método emergente para alcanzar la estabilidad hemodinámica de su hipertensión pulmonar persistente, el número de días de VMC fue de 34 y la estancia intrahospitalaria de 60 días, estos infantes evolucionaron con adecuado desarrollo neurológico y sólo una madre requirió de transfusión sanguínea perioperatoria, 2 pacientes fallecieron por sepsis y prematurez en uno y en otro por coagulopatía y síndrome de fuga aérea. ${ }^{1}$ Otra revisión importante demuestra el impacto de EXIT para la resección de estas masas fetales realizando un estudio comparativo entre 7 fetos obtenidos por parto convencional y 9 obtenidos por la vía de EXIT con resección pulmonar: el CVR promedio en ambos grupos fue de 2.6 con desviación mediastinal en todos los casos, con 37 SG en promedio, el tiempo EXIT fue de $65 \mathrm{~min}$; en el grupo convencional los 7 requirieron de una resección pulmonar de urgencia al momento de nacer por dificultad respiratoria y colapso hemodinámico y 2 de ellos murieron (mortalidad 71\%); en grupo EXIT con resección pulmonar la sobrevida fue del $100 \%$, los días de estancia hospitalaria y días de ventilación mecánica fueron de 10 y 3 días, respectivamente, para este último grupo EXIT. ${ }^{2}$ Nuestro caso correspondía a un feto con una masa pulmonar fetal de alto riesgo con un CVR persistente alto de 2.7 y franca desviación mediastinal con mal pronóstico y alta mortalidad al momento de nacer, durante el procedimiento EXIT se corroboran los impactos fisiológicos favorables de la descompresión mediastinal y cardiovascular de la toracotomía, así como el pinzamiento de la arteria pulmonar en la abolición del cortocircuito vascular-pulmonar. No obstante, consideramos que la causa de muerte en el posoperatorio inmediato correspondió al volu-barotrauma generado por la VMC en un pulmón residual hipoplásico con alta reactividad vascular que evolucionó al daño endotelial y a la hipertensión pulmonar persistente y refractaria al tratamiento ventilatorio y óxido nítrico; consideramos que la instauración temprana de una ventilación gentil como las estrategias de VAFO en el posquirúrgico inmediato podrían haber atenuado los efectos del barotrauma en un pulmón hipoplásico susceptible, la ECMO puede representar como en los grandes centros fetales una opción para la hipertensión pulmonar persistente refractaria a los manejos convencionales y sistemáticos, fue la evolución clínica agresiva y los hallazgos histopatológicos los que guardaron correlación con una MAQ sólida incompatible con la función y la vida.

Las MCBP pueden expresar otras repercusiones secundarias como en el caso del hidrotórax y quilotórax que complican los SBP, estos fetos podrían requerir durante el procedimiento EXIT la instauración de una toracocentesis evacuadora o la inserción de un drenaje pleural uni o bilateral que contribuyan a mejorar las condiciones hemodinámicas y de reexpansión pulmonar $y$, en consecuencia, favorecer la reanimación neonatal al momento del nacimiento. La asfixia y prematurez que podrían agregar morbimortalidad en estos fetos podrían requerir la aplicación de surfactante una vez estabilizada la vía aérea comprometida y el compromiso cardiopulmonar; una vía aérea comprometida podría requerir durante el procedimiento EXIT una laringoscopia directa e intentar la intubación endotraqueal, o bien en casos fallidos una intubación endotraqueal retrógrada o asistida por broncoscopia flexible, cuando es imposible la visualización laríngea quizás se requiera una 
broncoscopia rígida y potencial traqueostomía, algunas masas cervicales por la distorsión y compresión severa de la vía aérea podrán requerir de una resección durante el procedimiento EXIT. ${ }^{28-30}$

\section{CONCLUSIÓN}

El procedimiento EXIT podría ser considerado ante el diagnóstico prenatal de las masas torácicas fetales de alto riesgo que comprometan la vía aérea o pronostiquen inestabilidad cardiorrespiratoria y muerte al momento del nacimiento. Un equipo profesional multidisciplinario debe optimizar la perfusión úteroplacentaria que posibilite ejecutar los procedimientos endoscópicos y quirúrgicos para convertir una situación catastrófica e inestable en una situación controlada, una decisión colectiva, ética y rigurosa debe balancear los riesgos y beneficios del binomio candidato a EXIT.

\section{REFERENCIAS}

1. Hedrick HL, Flake AW, Crombleholme TM, et al. The ex utero intrapartum therapy procedure for high-risk fetal lung lesions. J Pediatr Surg 2005;40(6):1038-1044.

2. Cass DL, Olutoye $\mathrm{OO}, \mathrm{Cassady} \mathrm{Cl}$, et al. EXIT-to resection for fetuses with large lung masses and persistent mediastinal compression near birth. J Pediatr Surg 2013;48(1):138-144. doi: 10.1016/j.jpedsurg.2012.10.067.

3. Miniati DM, Chintagumpala M, Langston C, et al. Prenatal presentation and outcome of children with pleuropulmonary blastoma. J Pediatr Surg 2006;41(1):66-71.

4. Lazar DL, Cass DL, Dishop MK, et al. Fetal lung interstitial tumor: a cause of late gestation fetal hydrops. J Pediatr Surg 2011;46(6):1263-1266. doi: 10.1016/j. jpedsurg.2011.02.056.

5. Cass DL, Olutoye OO, Cassady $\mathrm{Cl}$, et al. Prenatal diagnosis and outcome of fetal lung masses. J Pediatr Surg 2011;46(2):292-298. doi: 10.1016/j.jpedsurg.2010.11.004.

6. Sancho HR, Solorio RL, Duran CA, et al. Secuestro pulmonar extralobar asintomático y la importancia del diagnóstico y tratamiento perinatal: el manejo expectante no es una opción terapéutica. Neumol Cir Torax 2015;74(4):262-270.

7. Adzick NS. Management of fetal lung lesions. Clin Perinatol 2009;36(2):363-376, x. doi: 10.1016/j. clp.2009.03.001.

8. Grethel EJ, Wagner AJ, Clifton MS, et al. Fetal intervention for mass lesions and hydrops improves outcome: a 15 year experience. J Pediatr Surg 2007;42 (1):117-123.

9. Curran PF, Jelin EB, Rand L, et al. Prenatal steroids for microcystic congenital cystic adenomatoid malformations. J Pediatr Surg 2010;45(1):145-150. doi: 10.1016/j. jpedsurg.2009.10.025.

10. Harrison MR, Adzick NS, Flake AW, et al. Correction of congenital diaphragmatic hernia in utero VIII: Response of the hypoplastic lung to tracheal occlusion. J Pediatr Surg 1996;31(10):1339-1348.

11. Marwan A, Crombleholme TM. The EXIT procedure: principles, pitfalls, and progress. Semin Pediatr Surg 2006;15(2):107-115.

12. Dighe MK, Peterson SE, Dubinsky TJ, Perkins J, Cheng E. EXIT procedure: technique and indications with prenatal imaging parameters for assessment of airway patency. Radiographics 2011;31(2):511-526. doi: 10.1148/ rg.312105108.

13. Thorper-Beeston JG, Nicolaides KH. Cystic adenomatoid malformation of the lung: prenatal diagnosis and outcome. Prenat Diagn 1994;14(8):677-688.

14. Adzick NS. Management of fetal lung lesions. Clin Perinatol 2003;30(3):481-492.

15. Adzick NS, Harrison MR, Glick PL, et al. Fetal cystic adenomatoid malformation: prenatal diagnosis and natural history. J Pediatr Surg 1985;20(5):483-488.

16. Adzick NS. Open fetal surgery for life-threatening fetal anomalies. Semin Fetal Neonatal Med 2010;15(1):1-8. doi: 10.1016/j.siny.2009.05.003.

17. Roggin KK, Breuer CK, Carr SR, et al. The unpredictable character of congenital cystic lung lesions. J Pediatr Surg 2000;35(5):801-805.

18. Cass DL, Crombleholme TM, Howell LJ, Stafford PW, Ruchelli ED, Adzick NS. Cystic lung lesions with systemic arterial blood supply: a hybrid of congenital cystic adenomatoid malformation and bronchopulmonary sequestration. J Peditr Surg 1997;32(7):986990.

19. Stanton M, Njere I, Ade-Ajayi N. Patel S, Davenport M. Systematic review and meta analysis of the postnatal management of congenital cystic lung lesions. J Pediatr Surg 2009;44(5):1027-1033. doi: 10.1016/j. jpedsurg.2008.10.118.

20. Durell J, Thakkar H, Gould S, Fowler D, Lakhoo K. Pathology of asymptomatic, prenatally diagnosed cystic lung malformations. J Pediatr Surg 2016;51(2):231-235. doi: 10.1016/j.jpedsurg.2015.10.061.

21. Crombleholme TM, Coleman B, Hedrick H, et al. Cystic adenomatoid malformation volume ratio predicts outcome in prenatally diagnosed cystic adenomatoid malformation of the lung. J Pediatr Surg 2002;37(3):331338.

22. Ehrenberg-Buchner S, Stapf AM, Berman DR, et al. Fetal lung lesions: can we start to breathe easier? Am J Obstet Gynecol 2013;208(2):151.e1-7. doi: 10.1016/j. ajog.2012.11.012.

23. Liechty KW. Ex utero intrapartum therapy. Semin Fetal Neonatal Med 2010;15(1):34-39. doi: 10.1016/j. siny.2009.05.007.

24. Marwan A, Crombleholme TM. The EXIT procedure: principles, pitfalls, and progress. Semin Pediatr Surg 2006;15(2):107-115.

25. Sancho HR, Solorio RL, Durán CA, Cuevas SFJ, López CE. Tratamiento perinatal de las malformaciones congénitas broncopulmonares en un país en vías de desarrollo: el punto de vista de la cirugía de tórax pediátrica [resumen]. Neumol Cir Torax 2015;74(1):72. 
26. Moldenhauer JS. Ex utero intrapartum therapy. Semin Pediatr Surg 2013;22(1):44-49. doi: 10.1053/j.sempedsurg. 2012.10.008.

27. Stoffan AP, Wilson JM, Jennings RW, Wilkins-Haug LE, Buchmiller TL. Does the ex utero intrapartum treatment to extracorporeal membrane oxygenation procedure change outcomes for high-risk patients with congenital diaphragmatic hernia? J Pediatr Surg 2012;47(6):10531057. doi: 10.1016/j.jpedsurg.2012.03.004.

28. Bouchard S, Johnson MP, Flake AW, et al. The EXIT procedure: experience and outcome in 31 cases. J Pediatr Surg 2002;37(3):418-426.

29. Hubbard AM, Crombleholme TM, Adzick NS. Prenatal MRI evaluation of giant neck masses in preparation for the fetal EXIT procedure. Am J Perinatol 1998; 15(4):253-257.
30. Prontera W, Jaeggi ET, Pfizenmaier M, Tassaux D, Pfister RE. Ex utero intrapartum treatment (EXIT) of severe fetal hydrothorax. Arch Dis Child Fetal Neonatal Ed 2002;86(1):F58-60.

\section{$\triangle$ Correspondencia:}

Dr. Rogelio Sancho-Hernández, Neumología y Cirugía de Tórax,

Instituto Nacional de Pediatría.

Avenida de los Insurgentes Sur, 3700-C, Coyoacán.

Colonia Insurgentes Cuicuilco, 04530,

Ciudad de México.

Correo electrónico: saherog@yahoo.com.mx

Los autores declaran no tener conflicto de intereses 